\title{
Simulating AtTention Disorder in Autistic PATIENTS BASED ON A COMPUTATIONAL MODEL WITH NEURAL NETWORKS REINFORCEMENT LEARNING APPROACH
}

\author{
Seyedeh Samaneh Seyedi ${ }^{1}$ and Abolfazl Darroudi ${ }^{2}$ \\ ${ }^{1}$ Department of Information Technology Management, \\ Alzahra University, Tehran, Iran \\ ${ }^{2}$ Department of Psychology and Educational Sciences, \\ Allameh Tabatabai University, Tehran, Iran
}

\begin{abstract}
Autism is an advanced neurological disease that affect communication and social behaviors, including attention -one of the fundamental skills to learn about the world around us. Autistic people have difficulty moving their attention from one point to another fluently. Due to the high prevalence of autism and its increasing progression, and the need to address common disorders in patients, this study aimed to implement and simulate a computational model for attention deficit disorder in autistic patients using MATLAB. This computational model has three components: context-sensitive reinforcement learning, contextual processing, and automation that can teach a shift-shift task automatically. At first, the model functions like normal people, but its performance gets closer to autistic people after changing a single parameter. This study demonstrates that even a simple computational model can be used for normal and abnormal developmental cases using a neural network reinforcement learning approach and provide valuable insights into autism.
\end{abstract}

\section{KEYWORDS}

Autism Spectrum Disorder (ASD), Attention Deficit, Shift Attention Task, Computational Model, Reinforcement Learning.

\section{INTRODUCTION}

Autism Spectrum Disorder (ASD) is a complex neurodevelopmental disorder whose etiology is unknown despite its prevalence $[1 ; 2]$. This disease is highly inheritable [3;4], and although there has been active research to discover the genetic factors and other biological symptoms of the disease $[5 ; 6 ; 7 ; 8]$, its diagnosis still depends solely on behavioral assessments [9]. Autism mainly affects men, so the rate of male-to-female infection is currently estimated at 4 to 1 [10].

ASD has a heterogeneous set of various social, cognitive, motor, and perceptual symptoms, including impaired social skills, isolation, speech and language disorders, imitation of a particular set of patterns, and attention to stereotypes. This heterogeneity makes it difficult to establish a comprehensive model for the disorder $[11 ; 12]$.

Studies examining the genetic and molecular basis of autism indicate more than 100 genes involved, many of which are also involved in synaptic development and function [13]. Hence, a renowned hypothesis is that autism results from a neurophysiologic excitation-to-inhibition (E/I) 
International Journal of Information Technology Convergence and Services (IJITCS)

Vol.11, No.1/2/3/4/5/6, December 2021

imbalance [14]. However, the relationship between this imbalance and the behavioral characteristics of autism remains unclear. Given the shared nature of autism and the poor correlation of symptoms [15], one possibility is that a large-scale imbalance has affected neurological calculations and caused widespread behavioral symptoms known as autism.

On the other hand, attention and related disorders that autistic people suffer from are raised today as an essential symptom of ASD. Normal people can focus on several objects simultaneously, while people with ASD pay only a lot of attention to one object and not to other objects. Attention disorders of an autistic person can cause him or her to have difficulty changing attention from one object to another $[10 ; 16]$.

Neural networks are composed of simple elements that act in parallel with each other and whose internal connections determine the function of the neural network primarily [17]. Therefore, neural network modeling can provide a good mapping of the actual functioning of the human brain - in particular, attention deficit - in autistic patients and be effective in understanding the problems they face and helping them recover. Using neural network simulations, we show that the behavior of normal and autistic people can be simulated in a single model. A computational perspective can help link our understanding of the genetic and molecular basis of autism to its behavioral characteristics and provide valuable insights into the disorder and possible treatments. Therefore, in the background section, an attempt has been made to discuss more the characteristics of ASD patients and examine this issue from different aspects. There is also information about neural networks and their modeling and how to teach and learn them. The reference model is introduced in the following, and its function and components are described and then simulated using MATLAB. This model simulates the attention function of autistic and normal people and makes it possible to compare them. Finally, we conclude and present the results of the simulations. In addition, using the experimental results we have from previous experiments, we compare the model's performance with actual samples.

\section{BACKGROUND}

\subsection{Autism}

Autism is a genetic disorder, and one of the five Pervasive Developmental Disorders (PDDs) emerges with disorders in social communication during the first three years of life. Autism, known for the advanced disorders it causes in the patient's social behaviors, leads to special interests and behaviors and has different degrees determined according to the degree of social communication disorders [18]. Autism is a prevalent disorder in the United States (reported for one in 54 children) and has become one of the most common neurological disorders in childhood [19].

Although the disease mainly demonstrates symptoms, i.e., communication disorders, people with autism have abilities such as accurate imitation of the behavior of others. Although they do not have the skills to do conceptual and innovative work with creativity, they are skilled at doing repetitive and clichéd tasks - such as math skills - that even ordinary people cannot do. Autistic people have a particular interest in the rules. They welcome the pursuit of ruled action. Their lives are intertwined with the uniformity of the situation, and any change in it causes intense emotional reactions in patients $[20 ; 16]$. In general, we can summarize the main symptoms of autism as follows:

1. Social disorders in people with autism are severe and persistent (chronic) and result from disorders in various parts of the nervous system. These sections are about perceiving and 
International Journal of Information Technology Convergence and Services (IJITCS)

Vol.11, No.1/2/3/4/5/6, December 2021

processing faces or expressing emotions and paying attention to the words, behaviors, and gestures. Although people with autism often suffer from a lack of social intelligence, they may have high general intelligence. They cannot take on some of the mental responsibilities that normal people do well. As autistic infant ages, social disorders become more pronounced, and the ability to make eye contact decreases [11]. An autistic person has problems expressing his feelings and difficulties meeting and interacting with new people in society. They distance themselves from other members of society and lose the ability to express empathy and sympathy $[21 ; 16]$.

2. Speech and language disorders: About half of all autistic patients have speech problems, which can be one of the first signs that parents notice autism in their children. On the other hand, people who acquire this skill speak very firmly and hard and use clichéd and repetitive words and sentences with different tones. Researchers classify people with autism as mild to severe, depending on the degree of impairment in their speech skills. Lack of this linguistic skill causes many problems in the lives of such people. For example, they gradually distance themselves from others and become isolated [16].

3. Limited Interests and Behaviors: In addition to the apparent disorders seen in the social and speech behaviors of people with autism, they sometimes show an unusual fascination with a particular object, person, or activity. They insist on the uniformity of conditions in their environment and engage in certain repetitive and specific regulatory behaviors. For example, an autistic child may experience emotional or physical distress as a new device enters the home or may tend to perform certain activities frequently throughout the day [22; $16 ; 11]$.

4. Attention Deficits: Attention is one of the essential abilities needed to learn about the world around us, and a defect in this area can partly explain the characteristics of cognitive development in people with autism. Attention is not a single function but has several parts, such as distracting from the current focus, shifting to the new focus and engaging in it, selecting related stimuli, and maintaining attention over time in the presence of distracting stimuli $[23 ; 18]$. People with autism have difficulty moving attention from one point to another, thus uniting different areas of interest into a whole [24]. The accuracy of people with autism compared to normal people on a particular issue is normal or even better, with more extended periods [16], but the problem is that when they focus on a problem, they cannot change their attention and adjust it to another subject. In general, neuroimaging studies have suggested three attention abnormalities among people with autism, as follows:

1) Releasing attention from a subject (body or person) is a reactionary function that occurs in the event of a series of unexpected stimuli. In this operation, the parietal cortex is a very active nervous part. People with autism who are deficient in the parietal cortex perform these procedures very slowly. The degree of deficiency of the parietal cortex is positively related to the extent of delay and slowness in changing attention [25].

2) In general, normal people in the event of a stimulus can predict the location of the stimulus and pay attention to it. If people with cerebellar tumors and people with autism cannot do so, their predictions are usually incorrect. They are very slow and careless in locating the stimulus [26].

3) The third abnormality in the attention of people with autism is Attention Shift Disorder. When a person's attention is focused on one stimulus, and suddenly another stimulus is triggered, a normal person immediately shifts his attention to the second stimulus, while 
International Journal of Information Technology Convergence and Services (IJITCS)

Vol.11, No.1/2/3/4/5/6, December 2021

a person with autism cannot do so. For example, when a mother talks to her autistic child and asks her child to look at a picture, her child cannot do so.

Other symptoms of autism include mimicry of specific behavioral patterns, inadequate emotional responses to many stimuli, and lack of adaptive time in motor behaviors [27].

\subsection{Modeling of Neural Networks}

Neural networks are composed of simple components called neurons that work in parallel and are inspired by biological neural networks. The function of the neural network is primarily determined by the connections that exist between the components. These neurons send information in the form of action potentials through guided links to other neurons. Based on knowledge about the function of biological neural networks, they can be modeled and imitated especially for learning ability [28]. Modeling neural networks requires a model that can acquire essential features of the nervous system and exhibit similar behavior. The goal of modeling is basically to create a simpler model of the system that maintains the general behavior of the system and helps to understand the system more efficiently. Proponents of neural network approaches argue that neural simulations operate at the intermediate level - between molecular and behavioral levels - and provide an opportunity to understand the causes of causing behaviors and the dynamics of the connecting network with molecular and genetic characteristics [16].

\subsubsection{Learning in Simple Neurons}

The primary role of a biological neuron is to sum up its inputs so that the sum of the inputs does not exceed a specific limit called the threshold and then to produce an output. Connecting neurons to each other may create networks that can do something, but they must be trained somehow to do something useful. What makes these models usable is their ability to learn [17]. We must increase the weighting coefficients when the neuron is active and otherwise reduce the coefficients to learn the network. In learning, only active inputs are affected because inactive inputs have no effect on the weighted sum, and changing them has no effect on that particular case and may disturb what has been learned so far. The mentioned points can be combined in the form of a single-layer perceptron algorithm as follows [29]:

$$
y(t)=f_{h}\left[\sum_{i=0}^{n} W_{i}(t) x_{i}(t)\right]
$$

Where $y(t)$ : Actual Output; $x_{0}:$ Threshold initial value ; $0 \leq i \leq n ; \theta$ :

Output Threshold value; $\mathrm{W}_{\mathrm{i}}(\mathrm{t})$ : The input weight $\mathrm{i}$ at time $\mathrm{t}$; and other weighting coefficients are obtained as follows:

$$
w_{i}(t+1)=w_{i}(t)+\eta x_{i}(t)
$$

If the actual output was zero and the optimal output was 1.

$$
w_{i}(t+1)=w_{i}(t)-\eta x_{i}(t)
$$

If the actual output is 1 and the desired output is zero.

Where $\eta$ is the positive recovery factor $(0 \leq \eta \leq 1)$ and controls the adjustment rate (correction) of weight coefficients. 
Widrow and Huff propose a similar algorithm (1960) called the Delta Rule. This rule calculates the difference between the weighted sum and the desired output value and calls it an error. Then the weighting coefficients are adjusted according to this error:

$$
\begin{gathered}
w_{i}(t+1)=w_{i}(t)+\eta \Delta x_{i}(t) \\
d(t)=\left\{\begin{array}{l}
+1 \\
-1
\end{array}\right.
\end{gathered}
$$

The delta error value can be written as follows, and is the difference between the desired system output and the actual output:

$$
\Delta=d(t)-y(t)
$$

This formula controls adding or subtracting weighting coefficients because if the desired output is 1 and the actual output is 0 , then the weighting coefficients increase.

\subsubsection{The Multi-Layer Perceptron Learning Rule}

The multi-layer perceptron learning rule is slightly more complex than the previous rule, called the general delta rule or the Back-Propagation Rule. The best way to understand this is to examine the network behavior when teaching the patterns offered. Random outputs are generated when a pattern is supplied to an untrained network. The error function shows that the difference between the actual and desired output must first be defined. This type of learning is called supervised because the desired output is specified. We can call the network training successful if its output gradually approaches the desired output; in other words, the error function must permanently be reduced. In order to achieve this goal, the weight coefficients of the communication lines of the units are modified using the general delta rule. The delta rule calculates the value of the error function and propagates it backward from one layer to the previous, so it is called Back-Propagation. The weighting coefficients of each unit are modified separately, thus reducing the error rate and training the network [17].

\subsubsection{Reinforcement Learning}

Reinforcement learning is an approach to artificial intelligence that, unlike classical approaches that downplay interactive learning, focuses on learning from an informed teacher or reasoning from a complete environmental model. In reinforcement learning, unlike most forms of machine learning, the learner is not told what to do [30]. One of the challenges that only occurs in reinforcement learning is the compromise between exploration and exploitation. To get high rewards, a reinforcement learning agent must choose actions that have been tried in the past and have effectively created rewards. However, to determine what these actions are, we should choose actions we have not tried before. The agent must use what he has already learned to reward and seek to improve the choice of actions in the future. The complicated puzzle exploits and explorations can be chosen exclusively without fail [31].

New reinforcement learning research uses two conventional frameworks for Marco Decision Processes (MDPs). In this framework, the agent and the environment interact in a chain of discrete time steps, $\mathrm{t}=0,1,2,3, \ldots$ in each step, the agent observes the environment in a state, $\mathrm{s}_{\mathrm{t}}$, and selects an action. In response, the environment creates a random state transition to a new state, $s_{t+1}$, and randomly issues a numerical reward, $r_{t+1}($ See Figure 1). In the long run, the agent seeks to maximize the reward received [32]. 


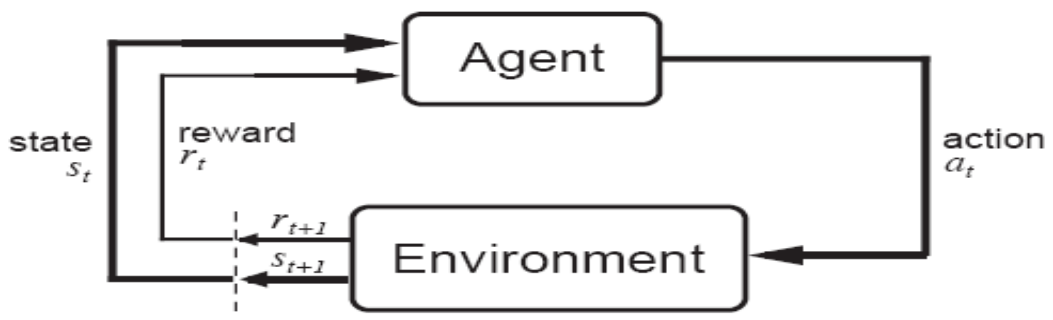

Figure 1. Reinforcement Learning Method [31]

The simplest reinforcement learning algorithms are applied directly to the agent experience and cause immediate interaction with the environment and behavior change. For example, tabular one-step Q-learning, one of the simplest reinforcement learning algorithms, uses the experiences of each state transition to modify an element of a table. With the symbol Q, this table has an input, $\mathrm{Q}(\mathrm{s}, \mathrm{a})$, for each pair of state, $\mathrm{s}$, and action, a. As soon as $\mathrm{s}_{\mathrm{t}} ; \mathrm{s}_{\mathrm{t}+1}$ passes the $\mathrm{a}_{\mathrm{t}}$ action and receives the $\mathrm{r}_{\mathrm{t}+1}$ reward, the following algorithm performs the following correction:

$Q\left(s_{t}, a_{t}\right) \leftarrow Q\left(s_{t}, a_{t}\right)+\alpha\left[r_{t+1}+\operatorname{\gamma max}_{a} Q\left(s_{t+1}, a_{t+1}\right)-Q\left(s_{t}, a_{t}\right)\right]$

Where $\alpha$ is a positive parameter with degree measurement, under the right conditions (ensuring adequate search and decreasing $\alpha$ over time), this process converges so that the greedy policy is optimal for Q; that is, in each case, s, an action, a, is selected for which $\mathrm{Q}(\mathrm{s}, \mathrm{a})$ has the most significant value. Therefore, this algorithm provides a way to find an optimal procedure directly and without any model of environmental dynamics. More sophisticated methods implement Q as a table and a trainable parameterized function such as an artificial neural network, so generalization between states is possible that can significantly reduce learning time and memory requirements [31].

\subsection{Attention Deficit Disorder}

Allport (1990) provided evidence against theories of attention, which assumed that sensory information should be selected at an early stage of processing. Instead, he suggested that the task of selective attention identifies factors for purposeful actions. To hold a particular object, one has to choose the coordinates of that object rather than other objects on the scene [33]. Many empirical studies support this view [34;35], indicating that an essential role of attention is to open the sensory channels of a particular action. Before reaching an object, it must first focus on the action-guiding information from the environment, so attentional fixations should be consistent with motor actions [36].

Focus attention is a way to seek information from the environment. In a series of experiments, Ballard et al. (1997) have shown that the subjects studied look briefly at the outside world, even when they remember the external state according to the truth. They reported that even for the simple act of copying a four-color block pattern, people stared at the model eighteen times, even though the pattern was easy to remember. Hence, humans prefer to use direct reasoning when information is obtained from the environment, even when the process is slower than the memorybased method [37].

The close relationship between attention and action raises the possibility that control of both is similarly helpful. Attention control has many similarities to movement control, which is evident not only in overt attention movements in the form of eye movement but also in other actions that do not require motor activity; In these cases, the action involves an inconspicuous shift of 
attention that only affects the way sensory information is processed. Evidence supporting the similarity of attention and action is condition-based and is provided by parts of the brain involved in both attention and motor control [36].

While attention is often directed to a stimulus, another critical aspect is that it diverts attention from irrelevant stimuli. In patients with parietal injury, one of the most noticeable effects of the disease is their ability to change the focus of attention from a stimulus. The prefrontal cortex causes another type of inhibition. Patients with injury to the forehead, especially in the orbital area, are easily distracted by external stimuli [38].

Fuster (1997) described this as an inability to deter attention from false stimuli. Injured animals in the orbital areas are unable to prevent a directional reaction and are usually hyperactive. Many experts have interpreted animal forehead hyperactivity as an expression of a tendency to overreact to external stimuli [39]. The aboveevidence offers a view of attention as encompassing at least two levels: The first level directs attention to environmental stimuli, and the second level is responsible for inhibitory control of the lower subsystem. Different types of learning are possible at both levels [36].

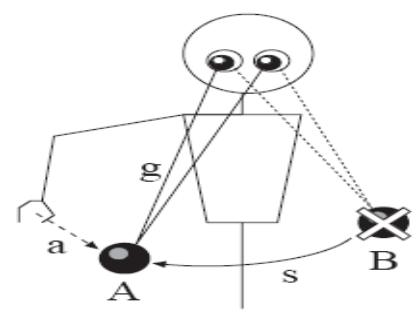

Figure 2. Four principles for attention [36]

Figure 2 describes the elements of an attention-controlled action. Attention is first focused on object $\mathrm{B}$, which frees the center of attention (attention as inhibition). Shifting attention and looking $\mathrm{g}$ from object $\mathrm{B}$ to $\mathrm{A}$ is an action $\mathrm{s}$ (attention as action). Object $\mathrm{B}$ is selected for action a (selection for action) by orienting the focus and staring at it. The focus of attention is used as an implicit argument for action. Without explicitly representing all its features, the focus of attention is referred to Object B (direct reasoning reference) and opens sensory channels is used as implicit reasoning for a motor action, which guides the hand to Object A [36].

Akshoomoff and Courchesne (1992) and Courchesne et al. (1994) tested eight children with autism diagnosed with cerebellar abnormalities. The purpose of this test was to examine the theory that the cerebellum helps to control the center of mental attention smoothly. Visual stimuli were green deflectors, and target light rays were red. Auditory stimuli, loud and slow, had frequencies of $2 \mathrm{kHz}$ and $1 \mathrm{kHz}$, respectively. The red light forced the person to press a key and acted as a stimulus to shift the focus to the auditory dimension. Similarly, the loudness of the target forced the subject to press a key and then shifted attention from the auditory to the visual dimension, regardless of the previous target dimension [40; 41].

The duration of visual and auditory stimuli was 50 milliseconds and the stimulation intervals varied between 450 and 1450 milliseconds. The target was used in the visual dimension as a sign to shift attention to the auditory dimension and vice versa. A person's performance was considered successful when he or she responded within 200 to 1400 milliseconds after displaying the target. With the same process as before, focused attention was also tested; Participants had to press a key by following a red light, regardless of auditory stimuli. 
International Journal of Information Technology Convergence and Services (IJITCS)

Vol.11, No.1/2/3/4/5/6, December 2021

Children with autism performed and typical children of the same age on their attention span but had difficulty moving accurately and quickly. When stimulation occurred 2.5 seconds or less after correct diagnosis of the previous target, children with autism could detect only $58.9 \%$ of targets, compared with normal children who correctly identified $78 \%$ of targets. However, over time, the performance of children with autism improved. This experiment's significant differences in normal children and children with autism make it an ideal test to develop a computational model for autism. The tasks are simple enough to implement in a computer simulation fully, and at the same time, the model describes many of the difficulties that people with autism face [40].

\section{A Computational Model Of Autism}

\subsection{The Purpose of the Model}

A computational architecture seeks to formulate the computational processes required for cognitive development explicitly. Such architectures' goal is also to understand cognitive development better or create control systems for robots [42]. To the extent that such schemes claim to describe or replicate human development, they should apply to developmental disorders such as autism. Therefore, a model is considered that can be used equally for normal and abnormal developmental cases, and it is believed that separate modeling of these cases is incorrect, and an acceptable model for autism should be based on a model of Have normal cognitive development [43].

Also, it should be possible to conduct face-to-face studies with the implemented model and compare its performance with that of normal people and people with developmental disorders. The simulated model in the present study shows how a unified design of multiple systems for reinforcement learning, contextual processing, and automation can learn test-based attention tasks for normal and autistic individuals and evaluate system parameters. It can be changed so that the model acts like a child with autism in attention. Although the simulated model is based on biological principles and replication of different brain areas, areas of the brain are not discussed in detail. The point is to understand the overall interaction between the components [43].

\subsection{Previous Models}

Many attempts have been made to develop computational models for autism. As far as we know, the first model in this field was created by Cohen (1994). Cohen believed that an insufficient number of hidden units in a back-propagation network could cause autism defects, as too many of these hidden units, compared to the activity's complexity, lead to habitual learning and poor generalization ability [43]. Gustafsson (1997) described autism as imperfect self-organization of feature mapping [44; 45]. He proposed his model based on Kohonen's (1995) self-organization maps, in which excessive inhibition leads to incomplete formation of cortical feature maps. A similar model was developed independently by de Carvalho et al. (1999) because autism arises from discrete and underdeveloped cortical maps. This model assumes that the initial amount of nerve-growth factor above the stimulus level (as in the Gustafen model) affects the formation of the map [46].

Another model based on cortical feature maps was proposed by Paplinski and Gustafsson (2002), who presented a simulation in which incomplete feature mappings were more due to familiaritybased preference than the likelihood of slightly noticeable shifts between stimuli. However, they concluded that preference was based on familiarity (choosing more of a small set of almost known stimuli) creates more assigned character mappings. It is perfectly correct, but the cause or 
effect of its abnormality is not clear. Also, it seems hasty to rule out attention deficits based on a single, straightforward model of attention in autism [45].

The disadvantage of such models is that they do not cause any behavior and, as a result, it is not possible to compare them with practical information. O'Loughlin and Thagard (2000) described another computational model that explains the lack of central coherence in autism. Their connectionist model is based on a network of constraint satisfaction [47], and it was believed that the lack of central cohesion is due to a very high level of inhibition compared to the stimulation level. This model is attractive in that it uses both normal and autistic cognition. This model also can apply to tests that are often performed on children with autism; Like the Sally-Ann task, in which the subject is shown a short game with two dolls named Sally and Ann who, by placing a bead in the basket and box, consider binary modes for action and belief [48]. However, Kamawar et al. (2002) believed this model predicts that autistic children will have problems with structurally incorrect image function, structurally similar to Sally-Ann's task; This identifies the main drawbacks of this model is the lack of a basis for learning or perception. However, there is no explanation as to how these nodes are formed [49].

This model also can apply to tests that are often performed on children with autism; like the falsebelief (Sally-Ann) task, in which the subject is shown a short game with two dolls named Sally and Ann who, by placing a marble in the basket and box, consider binary modes for action and belief [48]. However, according to Kamawar et al. (2002), this model predicts that autistic children will have problems with the false photo task structurally similar to Sally-Ann's task. Hence, this identifies the main drawbacks of this model - the lack of a basis for learning or perception: nodes with contexts such as "Sally thinks the marble is in the basket (but it is not)" are expressed, but no explanation is given about how these nodes are formed [49].

\subsection{The Reference Model}

This section describes a developmental model (Balkenius and Moren, 2004) to simulate the attention task [50]. This model is not considered a complete model of cognitive development; however, it contains crucial components that will be added to the complete structure. The model has three main parts (Figure 3). A Q-learning system learns the connections between stimuli and responses based on the received reinforcement (encouragement or punishment) [32]. The ContextQ algorithm used results from adding contextual inputs to the Q-learning algorithm [51].

A contextual system influences reinforcement learning to collect sensory inputs over time to create a code for the current context [36]. The contextual system acts as a working memory for the last potential target to which the system responds. Hence, the contextual Q-learning part can switch between different behavioral strategies depending on the current context. Unlike other reinforcement learning methods that can only learn a single policy, this allows for the gradual development of many different behaviors over time, a central feature of a developmental architecture.

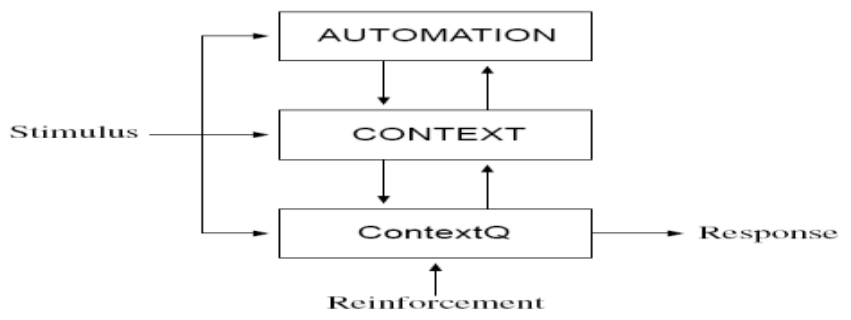

Figure 3. Overview of the Model [43] 
Finally, an automation system learns the connections between stimuli and contextual shifts, allowing the contextual system to change much faster when a target occurs. The role of the automation system is to create conditional contextual shifts due to the occurrence of a target stimulus, which accelerates the shifting between different tasks [43]. The three parts of the model are described in detail below.

\subsubsection{Context Processing}

Context: The concept of context encompasses many areas (See Figure 4). For example, a context could be a location specified by several specific symbols. The context can also be a chain of events or actions. In most cases, the context is temporal and spatial because the stimuli are usually deployed and must be considered. The context can include a single event in the finite sample, such as presenting a stimulus at distant times. In this case, the context necessarily acts as a stimulus rejection. A more exciting context occurs when the learning test itself is context. Context stimulation can even be produced internally, such as thought and feeling [50].
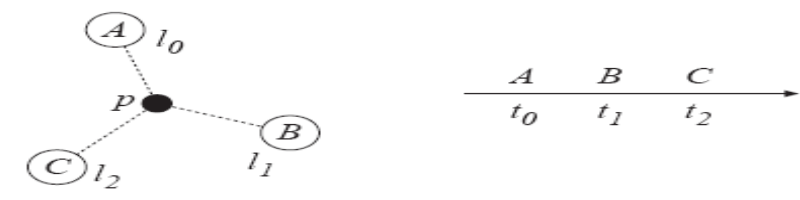

Figure 4. Two types of contexts. Left: A spatial context given by three stimuli at three locations. Right: A temporal context consisting of three events $\mathrm{A}, \mathrm{B}$, and $\mathrm{C}$.

Source: Balkenius, \& Morén (2000)

The Role of Context in Learning: Many types of learning are dependent on context. The relationship between context and memory is fascinating. While memory is often contextdependent, a temporally expanded context is very similar to memory. Donahoe and Palmer (1994) have suggested that working memory can be equated with adapting the sample to context. In adapting the sample, the subject should memorize an object and later match it to several stimuli present. Stimulation is memorized in the combined context and can later be used to control response accordingly. Another interesting relationship between context and attention is that attention can sometimes be seen as contextual separability, i.e., only one stimulus is considered, which is controlled by context [50].

\section{Contextual Processing Part of Model}

When a location $\mathrm{x}$ is considered inside the system or environment, the current input state of the attentional system - the focus of attention - is given by $\mathrm{s}(\mathrm{x})=\langle\mathrm{s} 0, \mathrm{~s} 1, \ldots, \mathrm{sn}\rangle$ which is a sensory code to stimulate attention in $\mathrm{x}$, and $\mathrm{L}(\mathrm{x})$ is also a code for its location $\mathrm{x}$. Given a set of attentional fixations $\mathrm{X} \subset \mathrm{V}$, a binding code $\mathrm{d}(\mathrm{X})$ is computed as the sum of the outer product of two vectors $\mathrm{s}(\mathrm{x})$ and $\mathrm{L}(\mathrm{x})$ for each location $\mathrm{x} \in \mathrm{X}[50]$ :

$$
d(x)=\sum_{x \in X}^{\infty}(\mathrm{s}(\mathrm{x}) \otimes L(x))
$$

The binding $d$ is a type of tensor coding of conjunctions as proposed by Smolensky (1999). Each binding code represents a context or a partial context. The output of the binding stage is integrated over time in a binding vector $\mathrm{b}=\langle\mathrm{b} 0, \mathrm{~b} 1, \ldots, \mathrm{bn}\rangle$. The following relation gives this vector: 
$b_{i}(t+1)=\frac{b_{i}(t)+\delta d_{i}+E_{i}}{\sum_{j=0}^{p}\left(b_{j}(t)+\delta d_{j}+E_{j}\right)}$

In other words, $b_{i}$ values add up their inputs over time, and the result is normalized to allow simple comparisons between the binding elements. The term $E_{i}$ is an additional input that will be explained below.

In previous models of contextual processing [50], individual binding codes are determined by an additional processing step - which gathers these codes as a contextual code. The current implementation does not include this step. Instead, the context output $c$ was set to the output of the binding nodes, $c_{i}=b_{i}$. Also, the location code is used in this model in part, using a single location that depends on the location of the response trigger, i.e., $\mathrm{L}(x)=\mathrm{a}_{0}$, where $\mathrm{a}_{0}$ is the system response as described below.

\subsubsection{Contextual Reinforcement Learning (ContextQ)}

ContextQ is used to learn the relationships between stimuli and responses in a context-dependent manner [51]. This algorithm can learn many distinct behaviors in different contexts and effectively generalize from one context to the next. Each state is expressed by a state vector $\mathrm{s}(\mathrm{x})$ $=\left\langle\mathrm{s}_{0}, \mathrm{~s}_{1}, \ldots, \mathrm{S}_{\mathrm{n}}\right\rangle$ and $\left\{\mathrm{a}_{0}, \mathrm{a}_{1}, \ldots, \mathrm{a}_{\mathrm{n}}\right\}$ is a discrete set of activities. The Q-function can be estimated as standard and as follows:

$Q\left(s, a_{j}\right)=\sum_{i=0}^{n} s_{i} w_{i j}$

Where the update rule is:

$\mathrm{w}_{\mathrm{ij}}(\mathrm{t}+1)=\mathrm{w}_{\mathrm{ij}}(\mathrm{t})+\alpha \mathrm{s}_{\mathrm{i}} \Delta \mathrm{Q}_{\mathrm{t}}$

$\mathrm{j}$ is the index of the last action and,

$\Delta \mathrm{Q}_{\mathrm{t}}=\left[\mathrm{r}_{\mathrm{t}+1}+\sigma \max _{\mathrm{a}} \mathrm{Q}\left(\mathrm{s}_{\mathrm{t}+1}, \mathrm{a}_{\mathrm{t}+1}\right)-\mathrm{Q}\left(\mathrm{s}_{\mathrm{t}}, \mathrm{a}_{\mathrm{t}}\right)\right]$

Each weight is updated based on the error value of the Q-function multiplied with the value of the state component $\mathrm{s}_{\mathrm{i}}$. That is, only components of the state that are related to the selected action will be updated. The linear approximator will generalize learning to similar states [31], which is the basic Q-learning algorithm described by [32]; contextual inputs will now be added to this algorithm

The context is described by the vector $\mathrm{c}=\left\langle\mathrm{c}_{0}, \mathrm{c}_{1}, \ldots, \mathrm{c}_{\mathrm{p}}\right\rangle$, which is received from the context system. Adding the contact to a linear approximator is done using the additional weights $\mathrm{u}_{\mathrm{ijk}}$, which link each association $\mathrm{w}_{\mathrm{ij}}$ to the context $\mathrm{c}_{\mathrm{k}}[43]$ :

$$
Q\left(c, s, a_{j}\right)=\sum_{i=0}^{n} s_{i} w_{i j} I_{i j}
$$


$I_{i j}=\prod_{k=0}^{p}\left(1-c_{k} u_{i j k}\right)$

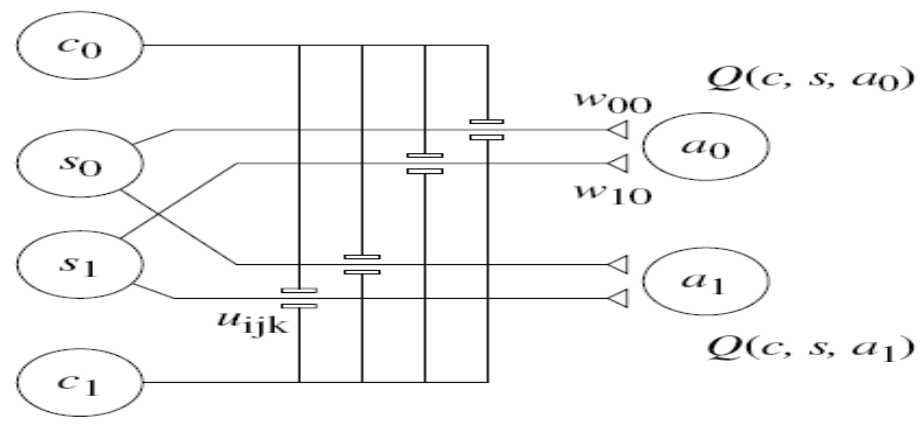

Figure 5. The approximation of $\mathrm{Q}(\mathrm{c}, \mathrm{s}, \mathrm{aj})$ as an artificial neural network with shunting inhibition from the context nodes ck to the association between a state node si and an action node aj [43]

In neural network terms, $I_{i j}$ can be seen as a propelling inhibition from the context of the association from the state to the action (See Figure 5). It is now examined how the learning rule should be changed to reflect the new context-sensitive estimator. The learning rule for the contextual weights is:

$$
\mathrm{u}_{\mathrm{ijk}}(\mathrm{t}+1)=\mathrm{u}_{\mathrm{ijk}}(\mathrm{t})-\beta \mathrm{s}_{\mathrm{i}} \mathrm{c}_{\mathrm{k}} \Delta \mathrm{Q}_{\mathrm{t}}
$$

Depending on the sign of $\Delta \mathrm{Q}_{\mathrm{t}}$, two different sets of learning constants are used. When $\Delta \mathrm{Q}_{\mathrm{t}}>0, \alpha=$ $\alpha^{+}$and $\beta=\beta^{+}$. Otherwise, $\alpha=\alpha^{-}$and $\beta=\beta^{-}$. Typically, $\alpha^{+}>\alpha^{-}$and $\beta^{+}<\beta^{-}$. In other words, inhibition of the current context mainly increases the association between the current state and the selected action when the actual reinforcement is less than expected.

In the simulation performed, only a single action is used, and the value of $\mathrm{Q}$ is considered the probability of a response occurring. The single-action will also be used as an input to the context system.

\subsubsection{Automation}

Automation is the learning of stimulus-response associations by observing the activities of another system. This learning is done so that these actions are created automatically without the system that initially created them. This process is similar to some forms of classical conditioning, such as that manifested in conditional eye-blinking responses [52]. Automation will be modeled in a fundamental way using two types of inputs and one output. Inputs $s=\left\langle\mathrm{S}_{\mathrm{o}}, \mathrm{s}_{1}, \ldots, \mathrm{S}_{\mathrm{q}}\right\rangle$ are related to output $\mathrm{y}$ through associative weights $\mathrm{v}_{\mathrm{ij}}$,

$$
y_{j}=\sum_{i=0}^{q} s_{i} v_{i j}
$$

The Delta rule (Widrow and Hoff, 1960 )is used to learn the target output T:

$$
\mathrm{v}_{\mathrm{ij}}(\mathrm{t}+1)=\mathrm{v}_{\mathrm{ij}}(\mathrm{t})+\varepsilon\left[\mathrm{T}_{\mathrm{j}}(\mathrm{t})-\mathrm{y}_{\mathrm{j}}(\mathrm{t}-\tau)\right] \mathrm{s}_{\mathrm{i}}(\mathrm{t}-\tau)
$$


International Journal of Information Technology Convergence and Services (IJITCS)

Vol.11, No.1/2/3/4/5/6, December 2021

The time difference $\tau$ is used to create the output of the automation system before the system it taps into, and $\varepsilon$ is the learning rate. Conditionally speaking, $\tau$ is the optimal time interval between two stimuli for conditioning.

The target in the automation system is to change the output of the context system [53], and the output of the automation module is sent to the context system through the additional input

$\mathrm{E}_{\mathrm{j}}(\mathrm{t})=\phi \mathrm{y}_{\mathrm{j}}(\mathrm{t})$.

$\mathrm{T}_{\mathrm{j}}(\mathrm{t})=\mathrm{c}_{\mathrm{j}}(\mathrm{t})-\mathrm{c}_{\mathrm{j}}(\mathrm{t}-1)$

\section{FINDINGS}

In this study, the task of attention shift previously described has been simulated [40; 41]. In these simulations, the following constants are used:

$\alpha^{+}=0.2, \alpha^{-}=0.1, \beta^{+}=0.1, \beta^{-}=0.2, \sigma=0, \delta=0.035, \varepsilon=0.01, \tau=2$.

The parameter $\phi$ is set to 50 for the normal group and 0 for the autism group. The duration of each simulation step is 50 milliseconds. Of these parameters, only two are essential for a response. The $\delta$ constant, which controls the speed of positional displacement, has been selected with the highest possible accuracy to recreate the response profile of autistic individuals. The constant $\varphi$, which describes the effect of the automation system on the situation, is also set to an appropriate value to produce a response similar to a normal group. When the model answers correctly, it receives a value of 1 (equivalent to encouragement), and for each incorrect answer, a value of 1- (equivalent to punishment) is assigned to the model. Reinforcement (Encouragement/punishment) is applied through a test.

It is necessary to mention a few points about performing simulations:

- In diagrams, the vertical and horizontal axes represent the $\mathrm{Q}$ function and the time steps, respectively.

- Due to the lack of main inputs of the model, random inputs have been used.

- In the simulations, six modes are considered.

- 1000 time steps have been used in performing the simulations.

- The vertical axis is multiplied by 5 so that the diagrams do not overlap.

- In these diagrams, the performance of the four neurons is shown in terms of their winning rate during the gradual training of the network, which shows the improvement in performance after training.

Figures 7 and 8 simulate the task of attention shift in two groups of autistic patients and normal people. Figures 9 and 10 also show the experimental data obtained from [40]. 
International Journal of Information Technology Convergence and Services (IJITCS)

Vol.11, No.1/2/3/4/5/6, December 2021

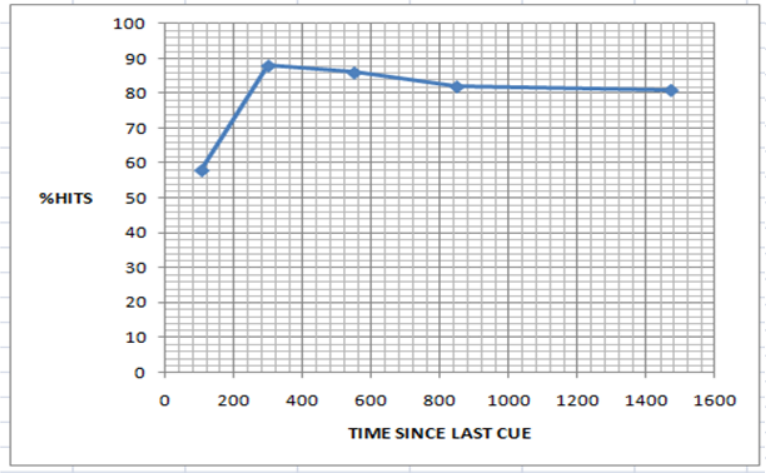

Figure 7. Simulation of attention shift task for the autistic group

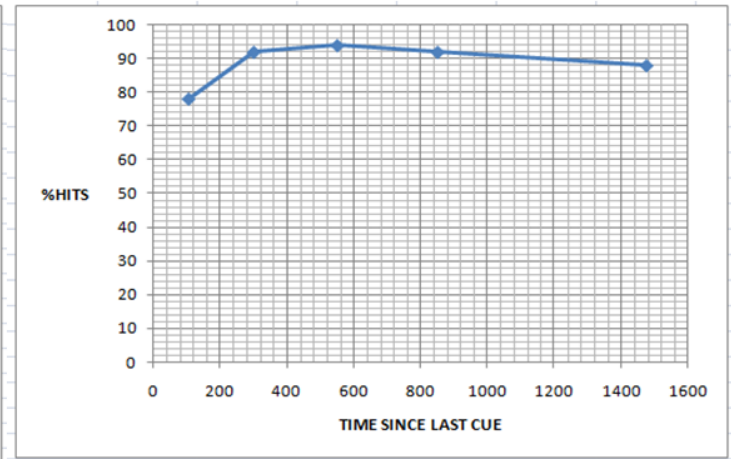

Figure 8. Simulation of attention shift task for the normal group

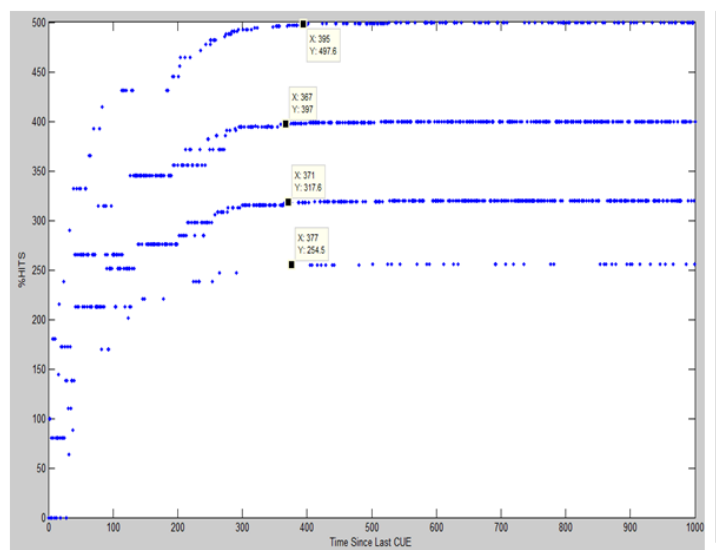

Figure 9. Experimental data from [40] for attention shift task among the normal groupattention

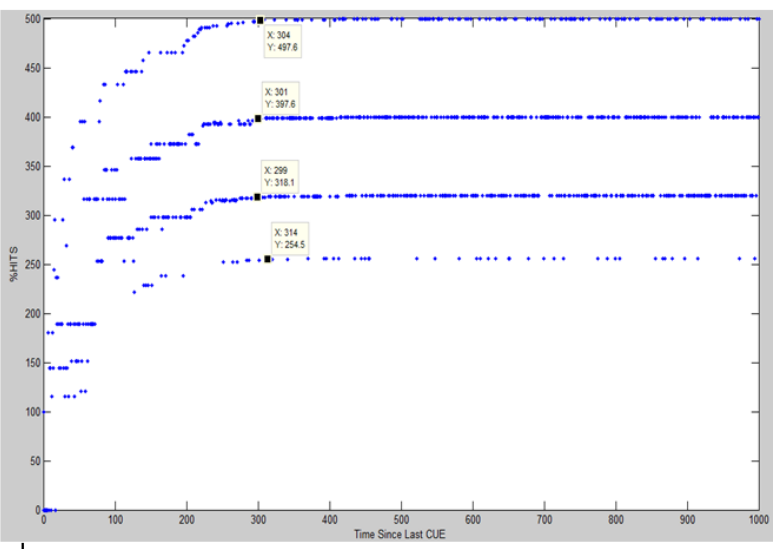

Figure 10. Experimental data from [40] for shift task among the autistic group

By performing the simulations, the following vital differences are obtained between the performance of the model for the normal group and the autism group:

1. In the case of the autistic group, the model achieves a stable state of functioning after a more extended period than the normal group.

2. In the autistic group, the upward trend in performance improvement is steeper than that of the normal group.

The simulations show that this computational model can recreate the significant behaviors of normal and autistic individuals in the attention shift task. For a comparison of experimental results and simulations, see Table 1.

Table 1- Experimental and Simulation Results in Normal vs. Autistic Group

\begin{tabular}{|l|l|l|}
\hline \multicolumn{1}{|c|}{ Normal Group } & \multicolumn{1}{c|}{ Autistic Group } \\
\hline $\begin{array}{l}\text { Experimental } \\
\text { Results }\end{array}$ & $\begin{array}{l}\text { It starts with about } 80 \% \text { of the test } \\
\text { performance and eventually } \\
\text { achieves performance at about } 90 \% .\end{array}$ & $\begin{array}{l}\text { It starts with performance of about } 60 \% \text { and } \\
\text { continues with an increasing trend and } \\
\text { achieves a final performance of } 80 \% .\end{array}$ \\
\hline $\begin{array}{l}\text { Simulation } \\
\text { Results }\end{array}$ & $\begin{array}{l}\text { It starts with a performance of } \\
\text { about } 20 \% \text { and ends with a final } \\
\text { value of } 100 \% .\end{array}$ & $\begin{array}{l}\text { It starts with a performance of about 15\% and } \\
\text { achieves a final performance of about 100\%, } \\
\text { with a process almost identical to the } \\
\text { simulation results. }\end{array}$ \\
\hline
\end{tabular}


International Journal of Information Technology Convergence and Services (IJITCS)

Vol.11, No.1/2/3/4/5/6, December 2021

There are three significant differences between experimental and simulation results:

1. The performance of the model in the simulation at the beginning of the second interval is worse than the autistic and normal groups

2. The final performance of the simulation is better than the experimental results.

3 . The trend of increasing the success of autistic people in the experiment is less than the model.

Since we do not have access to initial data from the work of [40; 41], it is not possible to statistically compare the model with experimental data. Therefore, it is not clear whether these slight differences in means are noticeable.

\section{DisCuSSION \& CONCLUSION}

One problem with autism research is that it is both genetically heterogeneous and typologically diverse. The present study investigated how computational models of neural networks can bridge the gap between analysis levels and genetic/molecular and behavioral findings. In particular, the simulated model shows how a computational system can learn to participate in a complex cognitive test based solely on the reinforcement (encouragement/punishment) received. This type of learning situation is usually categorized outside of the reinforcement learning models.

Importantly, computational frameworks similar to the present study can provide a systematic mechanism for generating falsifiable assumptions about the neural basis of autism. The three model subsystems can be related to the functional maps of brain structures [54]. The reinforcement learning system is similar to primary complexes. This system teaches reinforcement-sensitive stimulus-response associations. The contextual sensitivity of the model makes it one step ahead of other basal ganglia models, which usually do not have this additional input.

The automation system has a cerebellar-like function in that it learns stimulus-response association based on the repetition of a response after the occurrence of a specific stimulus independent of reinforcement. The context system is similar to the hippocampus and prefrontal cortex. As a result of these similarities with brain structures, the model links the two components of "autism as a problem in situational sensitivity and distraction" and "the suggested role of cerebellar dysfunction in autism." This conclusion is consistent with Skoyles's (2001) theory that autism is caused by a disconnection between the cerebellar cortex and the cerebellum.

The current model includes only the minimal implementations required for each subsystem to achieve the desired results. Interaction between subsystems is also minimal. In conclusion, this model for autism can be compared directly with experimental data from autistic individuals, and this model can reproduce the main differences between normal and autistic individuals in experimental practice. In addition, it can be demonstrated that the model can learn experimental operations without being specifically designed for them. Finally, it is concluded that the model's components are mapped to different brain structures, and it is suggested that disconnection of brain structures causes autism, which also disconnects subsystems leading to autistic behaviors.

The computational framework described in the present study provides a standard form for examining how neural computations affect autism symptoms. Applying this approach can provide insights into other mental disorders such as schizophrenia and possibly other old age aspects. The results of our simulations also suggest that behavioral experiments in combination with computational modeling can help identify different physiological pathways in individuals and facilitate the development of individual treatment programs. We suggest that computational perspectives can play an essential role in future mental health research and effectively provide insights to effectively understand and treat complex disorders such as autism. 


\section{REFERENCES}

[1] Levy SE., Mandell DS., \& Schultz RT., Autism, Lancet, 374(9701):1627-1638, 2009.

[2] McPartland J., \& Volkmar FR., Autism and related disorders, Handb Clin Neurol, 106:407-418, 2012.

[3] Folstein SE., \& Rosen-Sheidley B., Genetics of autism: complex aetiology for a heterogeneous disorder, Nat Rev Genet, 2(12):943-955, 2001.

[4] Geschwind DH., Advances in autism, Annu Rev Med, 60:367-380, 2009.

[5] Abrahams BS., \& Geschwind DH., Advances in autism genetics: on the threshold of a new neurobiology, Nat Rev Genet.,9(5):341-355, 2008.

[6] Scherer SW., and Dawson G., Risk factors for autism: translating genomic discoveries into diagnostics, Hum Genet.,30(1):123-148, 2011.

[7] Miles JH., Autism spectrum disorders--a genetics review, Genet Med., 278-294., 2011.

[8] Devlin B, \& Scherer SW., Genetic architecture in autism spectrum disorder, Curr Opin Genet Dev., 2012

[9] Huerta M, Lord C., Diagnostic evaluation of autism spectrum disorders. Pediatr Clin North Am., 2012

[10] Baron-Cohen S, Lombardo MV, Auyeung B, Ashwin E, Chakrabarti B, Knickmeyer R., Why are autism spectrum conditions more prevalent in males?, PLoS Biol, 2011

[11] American Psychiatric Association, Autism Spectrum Disorder, Available at: https://www.psychiatry.org/patients-families/autism/what-is-autism-spectrum-disorder, 2021.

[12] Rosenberg, A., Patterson, J. S., \& Angelaki, D. E., A computational perspective on autism, Proceedings of the National Academy of Sciences, 112(30), 9158-9165, 2015.

[13] Walsh CA., Morrow EM., \& Rubenstein JL., Autism and brain development, Cell 135(3):396-400, 2008.

[14] Rubenstein JL., \& Merzenich MM., Model of autism: Increased ratio of excitation/inhibition in key neural systems. Genes Brain Behav 2(5):255-267, 2003.

[15] Marko, M. K., Crocetti, D., Hulst, T., Donchin, O., Shadmehr, R., \& Mostofsky, S. H., Behavioural and neural basis of anomalous motor learning in children with autism, Brain, 138(3), 784-797, 2015.

[16] Galitsky, B., Computational Models of Autism, In Computational Autism, 17-77, Springer, Cham, 2016.

[17] Menhaj M.B., Basics of Neural Networks (Computational Intelligence), Amirkabir University Press. Volume 1. pp. 112-100. (Persian), 2008.

[18] Burack, J. A., Jenns, E. T., Stauder, J. E. A., Mottron, L., and Randolph, B., Attention and autism; behavioral and electrophysical evidence, In Cohen, D. J., and Volkmar, F. R. (Eds.), Handbook of autism and pervasive developmental disorders (pp. 226-247), New York: John Wiley \& Sons, Inc., 1997.

[19] Baio, J., Wiggins, L., Christensen, D. L., Maenner, M. J., Daniels, J., Warren, Z., \& Dowling, N. F., Prevalence of autism spectrum disorder among children aged 8 years - autism and developmental disabilities monitoring network, 11 sites, United States, 2014. MMWR Surveillance Summaries, 67(6), 1, 2018.

[20] Minshew NJ, Goldstein G., Autism as a disorder of complex information processing, Ment Retard Dev Disabil Res Rev 4:129-136, 1998.

[21] Mundy P, Sigman M, and Kasari C, A longitudinal study of joint attention and language development in autistic children, J Autism Dev Disord 20(1):115-128, 1990.

[22] Happe F, Ehlers S, Fletcher P, Frith U, Johansson M, and Gillberg C, Theory of mind in the brain. Evidence from a PET scan study of Asperger syndrome, Neuro Report 8:197-201, 1996.

[23] Hopfinger, J. B., Woldorff, M. G., Fletcher, E. M., Mangun, G. R., Dissociating top-down attentional control from selection perception and action, Neuropsychologia, 39, 1277-1291, 2001.

[24] Townsend, J., Courchesne, E., and Egaas, B., Slowed orienting of covert visual-spatial attention in autism: specific deficits associated with cerebellar and parietal abnormality, Development and Psychopathology, 8, 563-584, 1996.

[25] Konrad, K., \& Eickhoff, S. B., Is the ADHD brain wired differently? A review on structural and functional connectivity in attention deficit hyperactivity disorder, Human brain mapping, 31(6), 904-916, 2010.

[26] Stewart, H. J., McIntosh, R. D., \& Williams, J. H., A specific deficit of imitation in autism spectrum disorder, Autism Research, 6(6), 522-530, 2013. 
International Journal of Information Technology Convergence and Services (IJITCS)

Vol.11, No.1/2/3/4/5/6, December 2021

[27] Nadel, J., Perception-action coupling and imitation in autism spectrum disorder, Developmental Medicine \& Child Neurology, 57, 55-58, 2015.

[28] Kruse, R., Borgelt, C., Braune, C., Mostaghim, S., Steinbrecher, M., Klawonn, F., \& Moewes, C., Computational intelligence. Vieweg+ Teubner Verlag, 2011.

[29] Rosenblatt, F., Principles of neurodynamics. Perceptrons and the theory of brain mechanisms, Cornell Aeronautical Lab Inc Buffalo NY, 1961.

[30] Sutton, R. S., Introduction: The challenge of reinforcement learning, In Reinforcement Learning, Springer, Boston, MA, 1-3, 1992.

[31] Sutton, R. S., \& Barto, A. G., Reinforcement learning: An introduction. MIT press, 2018.

[32] Watkins, C. J. C. H., and Dayan, P., Q-learning. Machine Learning, 9, 279-292, 1992.

[33] Allport, A.,Visual attention. In M. Posner (Ed.), Foundations of Cognitive Science, Cambridge, MA: MIT Press, 631-682, 1990.

[34] Tipper, S. P., Howard, L. A., \& Houghton, G., Action-based mechanism of attention, Philos. 58. Trans. R. Soc. London Ser. B 353, 1385-1393, 1998.

[35] Frischen, A., Bayliss, A. P., \& Tipper, S. P., Gaze cueing of attention: Visual attention, social cognition, and individual differences, Psychological Bulletin, 133(4), 694, 2007.

[36] Balkenius, C., Attention, habituation, and conditioning: Toward a computational model, Cognitive Science Quarterly, 1(2), 171-214, 2000.

[37] Ballard, D. H., Hayhoe, M. M., Pook, P. K., \& Rao, R. P., Deictic codes for the embodiment of cognition, Behavioral and brain sciences, 20(4), 723-742, 1997.

[38] Rafal, R., \& Robertsson, L., In M. S. Gazzaniga (Ed.), The cognitive neurosciences, Cambridge, MA: MIT Press, 625-648,1995.

[39] Fuster, J. M., The prefrontal cortex: Anatomy, physiology, and neurophysiology of the frontal lobe, Philadelphia: Lippincott-Raven, 1997.

[40] Akshoomoff, N. A. and Courchesne, E., A new role for the cerebellum in cognitive operations, Behavioral Neuroscience, 106, 5, 731-738, 1992.

[41] Courchesne, E., Townsend, J., Akshoomoff, N. A., Saioh, O., Yeung-Courchesne, R., Lincoln, A. J., James, E. H., Haas, R. H., Schreibman, L., and Lau, L., Impairment in shifting attention in autistic and cerebellar patients, Behavioral Neuroscience, 108, 5, 848-865, 1994.

[42] Weng, J., and Zhang, Y., Developmental robots - a new paradigm. In Prince, C. G. Demiris, Y., Marom, Y., Kozima, H., and Balkenius, C. (Eds). Proceedings of the second international workshop on epigenetic robotics: modeling cognitive development in robotic systems, Lund University Cognitive Studies, 94, 2002.

[43] Balkenius, C., and Bjorne P., First steps toward a computational theory of autism, 27-34, 2004.

[44] Gustafsson, L., Inadequate cortical feature maps: a neural circuit theory of autism, Biological Psychiatry, 42, 1138-1147, 1997.

[45] Paplinski, A. P., and Gustafsson, L., An attempt in modeling autism using self-organizing maps, In Wang, L., Rajapakse, J. C., Fukushima, K., Lee, S-Y, and Yao, X. (Eds). Proceedings of the 9th International Conference on Neural Information Processing, 4, 2002.

[46] de Carvalho, L. A. V., de Carvalho Ferreira, N., and Fiszman, A., A Neurocomputational Model for Autism, Proceedings of IV Brazilian Conference on Neural Networks, 888-999, 1999.

[47] McClelland, J. L., and Rumelhart, D. E., Explorations in parallel distributed processing, Cambridge, MA: MIT Press, 1989.

[48] Wimmer H, \& Perner J., Beliefs about beliefs: representation and the containing function of wrong beliefs in young children's understanding of deception, Cognition 13:103-128, 1983.

[49] Kamawar, R., Garfield, J. L., and de Villiers, J., Coherence as an Explanation for Theory of Mind Task failure in Autism, Mind, and Language, 17, 3, 266-272, 2002

[50] Balkenius, C., \& Morén, J., A computational model of context processing, In 6th international conference on the simulation of adaptive behavior, 2000.

[51] Balkenius, C., \& Winberg, S., Cognitive modeling with context-sensitive reinforcement learning, Proceedings of AILS, 4, 10-19, 2004.

[52] Yeo, C., and Hesslow, Cerebellum and conditioned reflexes, Trends in Cognitive Science, 2, 9, 322331, 1998.

[53] Klopf, A. H., A neuronal model of classical conditioning. Psychobiology, 16, 85-125, 1988.

[54] Doya, K., Complementary roles of basal ganglia and cerebellum in learning and motor control, Current Opinion in Neurobiology, 10, 732-739, 2000. 